\title{
Some generalized Hermite-Hadamard type integral inequalities for generalized $s$-convex functions on fractal sets
}

\section{Adem Kılıçman ${ }^{1 *}$ and Wedad Saleh²}

\section{"Correspondence:}

akilic@upm.edu.my

'Department of Mathematics, Institute for Mathematical Research,

University Putra Malaysia, Serdang, Malaysia

Full list of author information is available at the end of the article

\begin{abstract}
In this article, some new integral inequalities of generalized Hermite-Hadamard type for generalized s-convex functions in the second sense on fractal sets have been established.
\end{abstract}

MSC: $26 \mathrm{~A} 51 ; 26 \mathrm{D} 07 ; 26 \mathrm{D} 15 ; 53 \mathrm{C} 22$

Keywords: $s$-convex functions; fractal space; local fractional derivative

\section{Introduction}

The convexity of functions is an important concept in the class mathematical analysis course, and it plays a significant role in many fields, for example, in biological system, economy, optimization, and so on [1-7]. Furthermore, there are a lot of several inequalities related to the class of convex functions. For example, Hermite-Hadamard's inequality is one of the well-known results in the literature, which can be stated as follows.

Theorem 1.1 (Hermite-Hadamard's inequality) Letf be a convex function on $\left[a_{1}, a_{2}\right]$ with $a_{1}<a_{2}$. If $f$ is integral on $\left[a_{1}, a_{2}\right]$, then

$$
f\left(\frac{a_{1}+a_{2}}{2}\right) \leq \frac{1}{a_{2}-a_{1}} \int_{a_{1}}^{a_{2}} f(x) d x \leq \frac{f\left(a_{1}\right)+f\left(a_{2}\right)}{2} .
$$

In [8], Dragomir and Fitzpatrick demonstrated a variation of Hadamard's inequality which holds for $s$-convex functions in the second sense.

Theorem 1.2 Let $f: \mathbb{R}_{+} \rightarrow \mathbb{R}_{+}$be an s-convex function in the second sense, $0<s<1$ and $a_{1}, a_{2} \in \mathbb{R}_{+}, a_{1}<a_{2}$. If $\in L^{1}\left(\left[a_{1}, a_{2}\right]\right)$, then

$$
2^{s-1} f\left(\frac{a_{1}+a_{2}}{2}\right) \leq \frac{1}{a_{2}-a_{1}} \int_{a_{1}}^{a_{2}} f(x) d x \leq \frac{f\left(a_{1}\right)+f\left(a_{2}\right)}{s+1} .
$$

In recent years, fractional calculus played an important part in fractal mathematics and engineering. In the sense of Mandelbrot, a fractal set is the one whose Hausdorff dimension strictly exceeds the topological dimension [9-15]. Many researchers studied the properties of functions on fractal space and constructed many kinds of fractional calculus by

(c) 2015 Kılıçman and Saleh. This article is distributed under the terms of the Creative Commons Attribution 4.0 International License (http://creativecommons.org/licenses/by/4.0/), which permits unrestricted use, distribution, and reproduction in any medium, provided you give appropriate credit to the original author(s) and the source, provide a link to the Creative Commons license, and indicate if changes were made. 
using different approaches [16-18]. Particularly, in [19], Yang stated the analysis of local fractional functions on fractal space systematically, which includes local fractional calculus and the monotonicity of function.

The outline of this article is as follows. In Section 2, we state the operations with real line number fractal sets and some definitions are given. Some integral inequalities of generalized Hermite-Hadamard type for generalized s-convex functions in the second sense are studied in Section 3. Finally, some applications are also illustrated in Section 4. The conclusions are in Section 5.

\section{Preliminaries}

Let $\mathbb{R}^{\alpha}$ be the real line numbers on fractal space. Then, by using Gao-Yang-Kang's concept, one can explain the definitions of the local fractional derivative and local fractional integral as in [19-23]. Now, if $r_{1}^{\alpha}, r_{2}^{\alpha}$ and $r_{3}^{\alpha} \in \mathbb{R}^{\alpha}(0<\alpha \leq 1)$, then

(1) $r_{1}^{\alpha}+r_{2}^{\alpha} \in \mathbb{R}^{\alpha}, r_{1}^{\alpha} r_{2}^{\alpha} \in \mathbb{R}^{\alpha}$,

(2) $r_{1}^{\alpha}+r_{2}^{\alpha}=r_{2}^{\alpha}+r_{1}^{\alpha}=\left(r_{1}+r_{2}\right)^{\alpha}=\left(r_{2}+r_{1}\right)^{\alpha}$,

(3) $r_{1}^{\alpha}+\left(r_{2}^{\alpha}+r_{3}^{\alpha}\right)=\left(r_{1}^{\alpha}+r_{2}^{\alpha}\right)+r_{3}^{\alpha}$,

(4) $r_{1}^{\alpha} r_{2}^{\alpha}=r_{2}^{\alpha} r_{1}^{\alpha}=\left(r_{1} r_{2}\right)^{\alpha}=\left(r_{2} r_{1}\right)^{\alpha}$,

(5) $r_{1}^{\alpha}\left(r_{2}^{\alpha} r_{3}^{\alpha}\right)=\left(r_{1}^{\alpha} r_{2}^{\alpha}\right) r_{3}^{\alpha}$,

(6) $r_{1}^{\alpha}\left(r_{2}^{\alpha}+r_{3}^{\alpha}\right)=\left(r_{1}^{\alpha} r_{2}^{\alpha}\right)+\left(r_{1}^{\alpha} r_{3}^{\alpha}\right)$,

(7) $r_{1}^{\alpha}+0^{\alpha}=0^{\alpha}+r_{1}^{\alpha}=r_{1}^{\alpha}$ and $r_{1}^{\alpha} \cdot 1^{\alpha}=1^{\alpha} \cdot r_{1}^{\alpha}=r_{1}^{\alpha}$.

Let us state some definitions about the local fractional calculus on $\mathbb{R}^{\alpha}$.

Definition 2.1 [19] A non-differentiable function $y: \mathbb{R} \rightarrow \mathbb{R}^{\alpha}$ is called local fractional continuous at $x_{0}$ if, for any $\varepsilon>0$, there exists $\delta>0$ such that

$$
\left|y(x)-y\left(x_{0}\right)\right|<\varepsilon^{\alpha}
$$

holds for $\left|x-x_{0}\right|<\delta$, where $\varepsilon, \delta \in \mathbb{R} . y \in C_{\alpha}\left(a_{1}, a_{2}\right)$ if it is local fractional continuous on the interval $\left(a_{1}, a_{2}\right)$.

Definition 2.2 [19] The local fractional derivative of $y(m)$ of order $\alpha$ at $m=m_{0}$ is defined by

$$
y^{\alpha}\left(m_{0}\right)=\left.\frac{d^{\alpha} y(m)}{d m^{\alpha}}\right|_{m=m_{0}}=\lim _{m \rightarrow m_{0}} \frac{\Gamma(1+\alpha)\left(y(m)-y\left(m_{0}\right)\right)}{\left(m-m_{0}\right)^{\alpha}}
$$

where $\Gamma(m)=\int_{0}^{\infty} m^{z-1} e^{-m} d m$. If there exists $y^{(n+1) \alpha}(m)=D_{m}^{\alpha} \cdots D_{m}^{\alpha} y(m)(n+1$ times $)$ for any $m \in I \subseteq \mathbb{R}$, then $y \in D_{(n+1) \alpha}(I), n=0,1,2, \ldots$.

Definition 2.3 [19] The local fractional integral of function $y(m)$ of order $\alpha$ is defined by, where $y \in C_{\alpha}\left[a_{1}, a_{2}\right]$,

$$
\begin{aligned}
a_{1} I_{a_{2}}^{(\alpha)} y(m) & =\frac{1}{\Gamma(1+\alpha)} \int_{a_{1}}^{a_{2}} y(t)(d t)^{\alpha} \\
& =\frac{1}{\Gamma(1+\alpha)} \lim _{\Delta t \rightarrow 0} \sum_{i=1}^{n} y\left(t_{i}\right)\left(\Delta t_{i}\right)^{\alpha}
\end{aligned}
$$


with $\triangle t_{i}=t_{i+1}-t_{i}$ and $\Delta t=\max \left\{\Delta t_{i}: i=1,2, \ldots, n-1\right\}$, where $\left[t_{i}, t_{i+1}\right], i=0,1, \ldots, n-1$ and $t_{0}=a_{1}<t_{1}<\cdots<t_{n-1}<t_{n}=a_{2}$ is a partition of the interval $\left[a_{1}, a_{2}\right]$.

In [24], the authors introduced the generalized convex function and established the generalized Hermite-Hadamard's inequality on fractal space. Let $f: I \subset \mathbb{R} \rightarrow \mathbb{R}^{\alpha}$ for any $x_{1}, x_{2} \in I$ and $\gamma \in[0,1]$ if the following inequality

$$
f\left(\gamma x_{1}+(1-\gamma) x_{2}\right) \leq \gamma^{\alpha} f\left(x_{1}\right)+(1-\gamma)^{\alpha} f\left(x_{2}\right)
$$

holds, then $f$ is called a generalized convex function on $I$. In $\alpha=1$, we have a convex function, convexity is defined only in geometrical terms as being the property of a function whose graph bears tangents only under it [25].

Theorem 2.1 (Generalized Hermite-Hadamard's inequality) Let $f \in{ }_{a_{1}} I_{a_{2}}^{(\alpha)}$ be a generalized convex function on $\left[a_{1}, a_{2}\right]$ with $a_{1}<a_{2}$. Then

$$
f\left(\frac{a_{1}+a_{2}}{2}\right) \leq \frac{\Gamma(1+\alpha)}{\left(a_{2}-a_{1}\right)^{\alpha}} a_{1} I_{a_{2}}^{(\alpha)} f(x) \leq \frac{f\left(a_{1}\right)+f\left(a_{2}\right)}{2^{\alpha}} .
$$

Note that it will be reduced to the class Hermite-Hadamard's inequality (1) if $\alpha=1$.

In [23], Mo and Sui introduced the definitions of two kinds of generalized $s$-convex functions on fractal sets as follows.

\section{Definition 2.4}

(i) A function $f: \mathbb{R}_{+} \rightarrow \mathbb{R}^{\alpha}$ is called generalized $s$-convex $(0<s<1)$ in the first sense if

$$
f\left(\gamma_{1} x_{1}+\gamma_{2} x_{2}\right) \leq \gamma_{1}^{s \alpha} f\left(x_{1}\right)+\gamma_{2}^{s \alpha} f\left(x_{2}\right)
$$

for all $x_{1}, x_{2} \in \mathbb{R}_{+}$and all $\gamma_{1}, \gamma_{2} \geq 0$ with $\gamma_{1}^{s}+\gamma_{2}^{s}=1$, we denote this class of functions by $G K_{s}^{1}$.

(ii) A function $f: \mathbb{R}_{+} \rightarrow \mathbb{R}^{\alpha}$ is called generalized $s$-convex $(0<s<1)$ in the second sense if inequality (3) holds for all $x_{1}, x_{2} \in \mathbb{R}_{+}$and all $\gamma_{1}, \gamma_{2} \geq 0$ with $\gamma_{1}+\gamma_{2}=1$, we denote this class of functions by $G K_{s}^{2}$.

In the same paper [23], Mo and Sui proved that all functions from $G K_{s}^{2}, s \in(0,1)$, are non-negative.

\section{Main results}

In [26], the authors demonstrated a variation of generalized Hadamard's inequality which holds for a generalized $s$-convex function in the second sense. Now, we will give another proof for generalized $s$-Hadamard's inequality.

Theorem 3.1 Let $f: \mathbb{R}_{+} \rightarrow \mathbb{R}_{+}^{\alpha}$ be a generalized s-convex function in the second sense, $0<$ $s<1$ and $a_{1}, a_{2} \in \mathbb{R}_{+}$with $a_{1}<a_{2}$. Iff $\in L^{1}\left(\left[a_{1}, a_{2}\right]\right)$, then

$$
\begin{aligned}
2^{\alpha(s-1)} f\left(\frac{a_{1}+a_{2}}{2}\right) & \leq \frac{\Gamma(1+\alpha)}{\left(a_{2}-a_{1}\right)^{\alpha}} a_{1} I_{a_{2}}^{(\alpha)} f(x) \\
& \leq \frac{\Gamma(1+s \alpha) \Gamma(1+\alpha)}{\Gamma(1+(s+1) \alpha)}\left(f\left(a_{1}\right)+f\left(a_{2}\right)\right) .
\end{aligned}
$$


Proof Since $f$ is generalized $s$-convex in the second sense, then

$$
f\left(\gamma a_{1}+(1-\gamma) a_{2}\right) \leq \gamma^{\alpha s} f\left(a_{1}\right)+(1-\gamma)^{\alpha s} f\left(a_{2}\right), \quad \forall \gamma \in[0,1] .
$$

Integrating the above inequality with respect to $\gamma$ on $[0,1]$, we have

$$
\begin{aligned}
\Gamma(1+\alpha)_{0} I_{1}^{(\alpha)} f\left(\gamma a_{1}+(1-\gamma) a_{2}\right) \leq & f\left(a_{1}\right) \Gamma(1+\alpha)_{0} I_{1}^{(\alpha)} \gamma^{\alpha s} \\
& +f\left(a_{2}\right) \Gamma(1+\alpha)_{0} I_{1}^{(\alpha)}(1-\gamma)^{\alpha s} \\
= & \frac{\Gamma(1+s \alpha) \Gamma(1+\alpha)}{\Gamma(1+(s+1) \alpha)}(f(a)+f(b)) .
\end{aligned}
$$

Let $x=\gamma a_{1}+(1-\gamma) a_{2}$. Then we have

$$
\begin{aligned}
\Gamma(1+\alpha)_{0} I_{1}^{(\alpha)} f\left(\gamma a_{1}+(1-\gamma) a_{2}\right) & =\frac{\Gamma(1+\alpha)}{\left(a_{1}-a_{2}\right)^{\alpha}} a_{2} I_{a_{1}}^{(\alpha)} f(x) \\
& =\frac{\Gamma(1+\alpha)}{\left(a_{2}-a_{1}\right)^{\alpha}} a_{1} I_{a_{2}}^{(\alpha)} f(x) .
\end{aligned}
$$

Now, it follows that

$$
\frac{\Gamma(1+\alpha)}{\left(a_{2}-a_{1}\right)^{\alpha}} a_{1} I_{a_{2}}^{(\alpha)} f(x) \leq \frac{\Gamma(1+s \alpha) \Gamma(1+\alpha)}{\Gamma(1+(s+1) \alpha)}\left(f\left(a_{1}\right)+f\left(a_{2}\right)\right)
$$

Then the second inequality in (4) is proved.

In order to prove the first inequality in (4), we use the following inequality:

$$
f\left(\frac{x_{1}+x_{2}}{2}\right) \leq \frac{f\left(x_{1}\right)+f\left(x_{2}\right)}{2^{\alpha s}}, \quad \forall x_{1}, x_{2} \in I
$$

Now, assume that $x_{1}=\gamma a_{1}+(1-\gamma) a_{2}$ and $x_{2}=(1-\gamma) a_{1}+\gamma a_{2}$ with $\gamma \in[0,1]$.

Then we get by inequality (5) that

$$
f\left(\frac{a_{1}+a_{2}}{2}\right) \leq \frac{f\left(\gamma a_{1}+(1-\gamma) a_{2}\right)+f\left((1-\gamma) a_{1}+\gamma a_{2}\right)}{2^{\alpha s}}, \quad \forall \gamma \in[0,1] .
$$

By integrating both sides of the above inequalities over $[0,1]$, we have

$$
\frac{1}{\Gamma(1+\alpha)} \int_{0}^{1} f\left(\frac{a_{1}+a_{2}}{2}\right)(d \gamma)^{\alpha} \leq \frac{1}{2^{\alpha(s-1)}\left(a_{2}-a_{1}\right)^{\alpha}} a_{1} I_{a_{2}}^{(\alpha)} f(x) .
$$

Then it follows that

$$
2^{\alpha(s-1)} f\left(\frac{a_{1}+a_{2}}{2}\right) \leq \frac{\Gamma(1+\alpha)}{\left(a_{2}-a_{1}\right)^{\alpha}} a_{1} I_{a_{2}}^{(\alpha)} f(x) .
$$

This completes the proof.

Remark 3.1 If we set $c=\frac{\Gamma(1+s \alpha) \Gamma(1+\alpha)}{\Gamma(1+(s+1) \alpha)}$ for $s \in(0,1]$, then it is best possible in the second inequality of (4). 
As the function $f:[0,1] \rightarrow\left[0^{\alpha}, 1^{\alpha}\right]$ given by $f(x)=x^{s \alpha}$ is generalized $s$-convex in the second sense,

$$
\begin{aligned}
\frac{\Gamma(1+\alpha)}{\left(a_{2}-a_{1}\right)^{\alpha}} a_{1} I_{a_{2}}^{(\alpha)} f(x) & =\Gamma(1+\alpha) \frac{1}{\Gamma(1+\alpha)} \int_{0}^{1} x^{\alpha s}(d x)^{\alpha} \\
& =\frac{\Gamma(1+s \alpha) \Gamma(1+\alpha)}{\Gamma(1+(s+1) \alpha)}
\end{aligned}
$$

and

$$
\frac{\Gamma(1+s \alpha) \Gamma(1+\alpha)}{\Gamma(1+(s+1) \alpha)}(f(0)+f(1))=\frac{\Gamma(1+s \alpha) \Gamma(1+\alpha)}{\Gamma(1+(s+1) \alpha)} .
$$

Similarly, if $\alpha=1$, then inequalities (4) reduce to inequalities (2).

Theorem 3.2 Let $A:[0,1] \rightarrow \mathbb{R}^{\alpha}$ be a function such as

$$
A(\gamma)=\frac{\Gamma(1+\alpha)}{\left(a_{2}-a_{1}\right)^{\alpha}} a_{1} I_{a_{2}}^{(\alpha)} f\left(\gamma x+(1-\gamma) \frac{a_{1}+a_{2}}{2}\right), \quad \gamma \in[0,1],
$$

where $f:\left[a_{1}, a_{2}\right] \rightarrow \mathbb{R}^{\alpha}$ is a generalized s-convex function in the second sense, $s \in(0,1]$, $a_{1}, a_{2} \in \mathbb{R}_{+}, a_{1}<a_{2}$ and $f \in L^{1}\left(\left[a_{1}, a_{2}\right]\right)$. Then

(i) $A \in G K_{s}^{2}$ on $[0,1]$,

(ii) we have the inequality

$$
A(\gamma) \geq 2^{\alpha(s-1)} f\left(\frac{a_{1}+a_{2}}{2}\right), \quad \forall \gamma \in[0,1]
$$

(iii) and the following inequality also holds:

$$
A \leq \min \left\{A_{1}(\gamma), A_{2}(\gamma)\right\}, \quad \gamma \in[0,1],
$$

where

$$
A_{1}(\gamma)=\gamma^{\alpha s} \frac{\Gamma(1+\alpha)}{\left(a_{2}-a_{1}\right)^{\alpha}} a_{1} I_{a_{2}}^{(\alpha)} f(x)+(1-\gamma)^{\alpha s} f\left(\frac{a_{1}+a_{2}}{2}\right)
$$

and

$$
\begin{aligned}
A_{2}(\gamma)= & \frac{\Gamma(1+\alpha s) \Gamma(1+\alpha)}{\Gamma(1+(s+1) \alpha)}\left(f\left(\gamma a_{1}+(1-\gamma) \frac{a_{1}+a_{2}}{2}\right)\right. \\
& \left.+f\left(\gamma a_{2}+(1-\gamma) \frac{a_{1}+a_{2}}{2}\right)\right)
\end{aligned}
$$

for $\gamma \in(0,1]$.

(iv) If $\tilde{A}=\max \left\{A_{1}(\gamma), A_{2}(\gamma)\right\}, \gamma \in[0,1]$, then

$$
\tilde{A} \leq \frac{\Gamma(1+\alpha s) \Gamma(1+\alpha)}{\Gamma(1+(s+1) \alpha)}\left\{\gamma^{\alpha s}\left(f\left(a_{1}\right)+f\left(a_{2}\right)\right)+2^{\alpha}(1-\gamma)^{\alpha s} f\left(\frac{a_{1}+a_{2}}{2}\right)\right\} .
$$


Proof (i) Let $\gamma_{1}, \gamma_{2} \in[0,1]$ and $\mu_{1}, \mu_{2} \geq 0$ with $\mu_{1}+\mu_{2}=1$, then

$$
\begin{aligned}
A\left(\mu_{1} \gamma_{1}+\mu_{2} \gamma_{2}\right)= & \frac{\Gamma(1+\alpha)}{\left(a_{2}-a_{1}\right)^{\alpha}} a_{1} I_{a_{2}}^{(\alpha)} f\left(\left(\mu_{1} \gamma_{1}+\mu_{2} \gamma_{2}\right) x+\left(1-\left(\mu_{1} \gamma_{1}+\mu_{2} \gamma_{2}\right)\right) \frac{a_{1}+a_{2}}{2}\right) \\
\leq & \frac{\Gamma(1+\alpha)}{\left(a_{2}-a_{1}\right)^{\alpha}} a_{1} I_{a_{2}}^{(\alpha)}\left\{\mu_{1}^{\alpha s} f\left(\gamma_{1} x+\left(1-\gamma_{1}\right) \frac{a_{1}+a_{2}}{2}\right)\right. \\
& \left.+\mu_{2}^{\alpha s} f\left(\gamma_{2} x+\left(1-\gamma_{2}\right) \frac{a_{1}+a_{2}}{2}\right)\right\} \\
= & \mu_{1}^{\alpha s} A\left(\gamma_{1}\right)+\mu_{2}^{\alpha s} A\left(\gamma_{2}\right),
\end{aligned}
$$

which implies that $A \in G K_{s}^{2}$ on $[0,1]$.

(ii) Let $\gamma \in(0,1]$ and by the change of variable $m=\gamma x+(1-\gamma) \frac{a_{1}+a_{2}}{2}$, we have

$$
A(\gamma)=\frac{\Gamma(1+\alpha)}{\gamma^{\alpha}\left(a_{2}-a_{1}\right)^{\alpha} \gamma a_{1}+(1-\gamma) \frac{a_{1}+a_{2}}{2}} I_{\gamma a_{2}+(1-\gamma) \frac{a_{1}+a_{2}}{2}}^{(\alpha)} f(m)=\frac{\Gamma(1+\alpha)}{\left(b_{2}-b_{1}\right)^{\alpha}} b_{1} I_{b_{2}}^{(\alpha)} f(m) .
$$

By using the first generalized Hermite-Hadamard inequality, we have

$$
\frac{\Gamma(1+\alpha)}{\left(b_{2}-b_{1}\right)^{\alpha}} b_{1} I_{b_{2}}^{(\alpha)} f(m) \geq 2^{\alpha(s-1)} f\left(\frac{b_{1}+b_{2}}{2}\right)=2^{\alpha(s-1)} f\left(\frac{a_{1}+a_{2}}{2}\right),
$$

and inequality (6) is obtained.

If $\gamma=0$, the inequality

$$
f\left(\frac{a_{1}+a_{2}}{2}\right) \geq 2^{\alpha(s-1)} f\left(\frac{a_{1}+a_{2}}{2}\right)
$$

also holds.

(iii) By using the second part of generalized Hadamard's inequality, we get

$$
\begin{aligned}
\frac{\Gamma(1+\alpha)}{\left(b_{2}-b_{1}\right)^{\alpha} b_{1}} I_{b_{2}}^{(\alpha)} f(m) \leq & \frac{\Gamma(1+s \alpha) \Gamma(1+\alpha)}{\Gamma(1+(s+1) \alpha)}\left(f\left(b_{1}\right)+f\left(b_{2}\right)\right) \\
= & \frac{\Gamma(1+s \alpha) \Gamma(1+\alpha)}{\Gamma(1+(s+1) \alpha)}\left(f\left(\gamma a_{1}+(1-\gamma) \frac{a_{1}+a_{2}}{2}\right)\right. \\
& \left.+f\left(\gamma a_{2}+(1-\gamma) \frac{a_{1}+a_{2}}{2}\right)\right) \\
= & A_{2}(\gamma), \quad \forall \gamma \in[0,1] .
\end{aligned}
$$

If $\gamma=0$, then the inequality

$$
f\left(\frac{a_{1}+a_{2}}{2}\right)=A(0) \leq A_{2}(0)=\frac{2^{\alpha} \Gamma(1+s \alpha) \Gamma(1+\alpha)}{\Gamma(1+(s+1) \alpha)} f\left(\frac{a_{1}+a_{2}}{2}\right)
$$

holds as it is equivalent to

$$
\left(\frac{\Gamma(1+(s+1) \alpha)}{\Gamma(1+s \alpha) \Gamma(1+\alpha)}-2^{\alpha}\right) f\left(\frac{a_{1}+a_{2}}{2}\right) \leq 0^{\alpha}
$$


and we know that for $s \in(0,1)$,

$$
f\left(\frac{a_{1}+a_{2}}{2}\right) \geq 0^{\alpha}
$$

Since

$$
f\left(\gamma x+(1-\gamma) \frac{a_{1}+a_{2}}{2}\right) \leq \gamma^{\alpha s} f(x)+(1-\gamma)^{\alpha s} f\left(\frac{a_{1}+a_{2}}{2}\right)
$$

for $\forall \gamma \in[0,1]$ and $x \in\left[a_{1}, a_{2}\right]$, then we obtain

$$
\begin{aligned}
A(\gamma) & =\frac{\Gamma(1+\alpha)}{\left(a_{2}-a_{1}\right)^{\alpha}} a_{1} I_{a_{2}}^{(\alpha)} f\left(\gamma x+(1-\gamma) \frac{a_{1}+a_{2}}{2}\right) \\
& \leq \gamma^{\alpha s} \frac{\Gamma(1+\alpha)}{\left(a_{2}-a_{1}\right)^{\alpha}} a_{1} I_{a_{2}}^{(\alpha)} f(x)+(1-\gamma)^{\alpha s} f\left(\frac{a_{1}+a_{2}}{2}\right) \\
& =A_{1}(\gamma) .
\end{aligned}
$$

Then, the proof of inequality (7) is complete.

(iv) We have

$$
\begin{aligned}
A_{2}(\gamma)= & \frac{\Gamma(1+s \alpha) \Gamma(1+\alpha)}{\Gamma(1+(s+1) \alpha)}\left[f\left(\gamma a_{1}+(1-\gamma) \frac{a_{1}+a_{2}}{2}\right)+f\left(\gamma a_{2}+(1-\gamma) \frac{a_{1}+a_{2}}{2}\right)\right] \\
\leq & \frac{\Gamma(1+s \alpha) \Gamma(1+\alpha)}{\Gamma(1+(s+1) \alpha)}\left[\gamma^{\alpha s} f\left(a_{1}\right)+(1-\gamma)^{\alpha s} f\left(\frac{a_{1}+a_{2}}{2}\right)\right. \\
& \left.+\gamma^{\alpha s} f\left(a_{2}\right)+(1-\gamma)^{\alpha s} f\left(\frac{a_{1}+a_{2}}{2}\right)\right] \\
= & \frac{\Gamma(1+s \alpha) \Gamma(1+\alpha)}{\Gamma(1+(s+1) \alpha)}\left[\gamma^{\alpha s}\left(f\left(a_{1}\right)+f\left(a_{2}\right)\right)+2^{\alpha}(1-\gamma)^{\alpha s} f\left(\frac{a_{1}+a_{2}}{2}\right)\right], \\
& \forall \gamma \in[0,1] .
\end{aligned}
$$

Since

$$
\frac{\Gamma(1+\alpha)}{\left(a_{2}-a_{1}\right)^{\alpha}} a_{1} I_{a_{2}}^{(\alpha)} f(x) \leq \frac{\Gamma(1+s \alpha) \Gamma(1+\alpha)}{\Gamma(1+(s+1) \alpha)}\left(f\left(a_{1}\right)+f\left(a_{2}\right)\right)
$$

and

$$
(1-\gamma)^{\alpha s} f\left(\frac{a_{1}+a_{2}}{2}\right) \leq 2^{\alpha}(1-\gamma)^{\alpha s} \frac{\Gamma(1+s \alpha) \Gamma(1+\alpha)}{\Gamma(1+(s+1) \alpha)} f\left(\frac{a_{1}+a_{2}}{2}\right)
$$

then

$$
\begin{aligned}
A_{1}(\gamma) \leq & \gamma^{\alpha s} \frac{\Gamma(1+s \alpha) \Gamma(1+\alpha)}{\Gamma(1+(s+1) \alpha)}\left(f\left(a_{1}\right)+f\left(a_{2}\right)\right) \\
& +2^{\alpha}(1-\gamma)^{\alpha s} \frac{\Gamma(1+s \alpha) \Gamma(1+\alpha)}{\Gamma(1+(s+1) \alpha)} f\left(\frac{a_{1}+a_{2}}{2}\right)
\end{aligned}
$$

and the proof of Theorem 3.2 is complete. 
Remark 3.2 In particular:

1. If we choose $s=1$ in Theorem 3.2, then we get:

(a)

$$
\begin{aligned}
& \frac{\Gamma(1+\alpha)}{\left(a_{2}-a_{1}\right)^{\alpha} a_{1}} I_{a_{2}}^{(\alpha)} f\left(\gamma x+(1-\gamma) \frac{a_{1}+a_{2}}{2}\right) \\
& \leq \min \left\{\gamma^{\alpha} \frac{\Gamma(1+\alpha)}{\left(a_{2}-a_{1}\right)^{\alpha}} a_{1} I_{a_{2}}^{(\alpha)} f(x)+(1-\gamma)^{\alpha} f\left(\frac{a_{1}+a_{2}}{2}\right)\right. \\
& \quad \frac{(\Gamma(1+\alpha))^{2}}{\Gamma(1+2 \alpha)}\left(f\left(\gamma a_{1}+(1-\gamma) \frac{a_{1}+a_{2}}{2}\right)\right. \\
& \left.\left.+f\left(\gamma a_{2}+(1-\gamma) \frac{a_{2}+a_{2}}{2}\right)\right)\right\} .
\end{aligned}
$$

(b) Since

$$
\begin{aligned}
\tilde{A}= & \max \left\{\gamma^{\alpha} \frac{\Gamma(1+\alpha)}{\left(a_{2}-a_{1}\right)^{\alpha}} a_{1} I_{a_{2}}^{(\alpha)} f(x)+(1-\gamma)^{\alpha} f\left(\frac{a_{1}+a_{2}}{2}\right),\right. \\
& \left.\frac{(\Gamma(1+\alpha))^{2}}{\Gamma(1+2 \alpha)}\left(f\left(\gamma a_{1}+(1-\gamma) \frac{a_{1}+a_{2}}{2}\right)+f\left(\gamma a_{2}+(1-\gamma) \frac{a_{1}+a_{2}}{2}\right)\right)\right\},
\end{aligned}
$$

we have

$$
\tilde{A}(\gamma) \leq \frac{(\Gamma(1+\alpha))^{2}}{\Gamma(1+2 \alpha)}\left[\gamma^{\alpha}\left(f\left(a_{1}\right)+f\left(a_{2}\right)\right)+2^{\alpha}(1-\gamma)^{\alpha} f\left(\frac{a_{1}+a_{2}}{2}\right)\right]
$$

2. Now if one chooses $\alpha=1$ in Theorem 3.2, then we can easily obtain:

(a)

$$
\begin{aligned}
& \frac{1}{\left(a_{2}-a_{1}\right)} \int_{a_{1}}^{a_{2}} f\left(\gamma x+(1-\gamma) \frac{a_{1}+a_{2}}{2}\right) d x \\
& \leq \min \left\{\gamma^{s} \frac{1}{\left(a_{2}-a_{1}\right)} \int_{a_{1}}^{a_{2}} f(x) d x+(1-\gamma)^{s} f\left(\frac{a_{2}+a_{2}}{2}\right)\right. \\
& \left.\quad \frac{1}{s+1}\left(f\left(\gamma a_{1}+(1-\gamma) \frac{a_{1}+a_{2}}{2}\right)+f\left(\gamma a_{2}+(1-\gamma) \frac{a_{1}+a_{2}}{2}\right)\right)\right\}
\end{aligned}
$$

(b) Similarly we have

$$
\begin{aligned}
\tilde{A}= & \max \left\{\gamma^{s} \frac{1}{\left(a_{2}-a_{1}\right)} \int_{a_{1}}^{a_{2}} f(x) d x+(1-\gamma)^{s} f\left(\frac{a_{1}+a_{2}}{2}\right),\right. \\
& \left.\frac{1}{s+1}\left(f\left(\gamma a_{1}+(1-\gamma) \frac{a_{1}+a_{2}}{2}\right)+f\left(\gamma a_{2}+(1-\gamma) \frac{a_{1}+a_{2}}{2}\right)\right)\right\}
\end{aligned}
$$

and

$$
\tilde{A}(\gamma) \leq \frac{1}{s+1}\left[\gamma^{s}\left(f\left(a_{1}\right)+f\left(a_{2}\right)\right)+2(1-\gamma)^{s} f\left(\frac{a_{2}+a_{2}}{2}\right)\right] \text { for } \forall \gamma \in[0,1]
$$

3. If one considers $\alpha=1$ and $s=1$ in Theorem 3.2, then we get: 
(a)

$$
\begin{aligned}
& \frac{1}{\left(a_{2}-a_{1}\right)} \int_{a_{1}}^{a_{2}} f\left(\gamma x+(1-\gamma) \frac{a_{1}+a_{2}}{2}\right) d x \\
& \leq \min \left\{\gamma \frac{1}{\left(a_{2}-a_{1}\right)} \int_{a_{1}}^{a_{2}} f(x) d x+(1-\gamma) f\left(\frac{a_{2}+a_{2}}{2}\right),\right. \\
& \left.\quad \frac{1}{2}\left(f\left(\gamma a_{1}+(1-\gamma) \frac{a_{1}+a_{2}}{2}\right)+f\left(\gamma a_{2}+(1-\gamma) \frac{a_{1}+a_{2}}{2}\right)\right)\right\} .
\end{aligned}
$$

(b)

$$
\begin{aligned}
\tilde{A}= & \max \left\{\gamma \frac{1}{\left(a_{2}-a_{1}\right)} \int_{a_{1}}^{a_{2}} f(x) d x+(1-\gamma) f\left(\frac{a_{1}+a_{2}}{2}\right),\right. \\
& \left.\frac{1}{2}\left(f\left(\gamma a_{1}+(1-\gamma) \frac{a_{1}+a_{2}}{2}\right)+f\left(\gamma a_{2}+(1-\gamma) \frac{a_{1}+a_{2}}{2}\right)\right)\right\}
\end{aligned}
$$

and

$$
\tilde{A}(\gamma) \leq \frac{1}{2}\left[\gamma\left(f\left(a_{1}\right)+f\left(a_{2}\right)\right)+2(1-\gamma) f\left(\frac{a_{2}+a_{2}}{2}\right)\right] \text { for } \forall \gamma \in[0,1] .
$$

Theorem 3.3 Let $g:[0,1] \rightarrow \mathbb{R}^{\alpha}$ be a function such as

$$
g(\gamma)=\frac{1}{(\Gamma(1+\alpha))^{2}} \frac{1}{\left(a_{2}-a_{1}\right)^{2 \alpha}} \int_{a_{1}}^{a_{2}} \int_{a_{1}}^{a_{2}} f\left(\gamma x_{1}+(1-\gamma) x_{2}\right)\left(d x_{1}\right)^{\alpha}\left(d x_{2}\right)^{\alpha}, \quad \gamma \in[0,1]
$$

where $f:\left[a_{1}, a_{2}\right] \rightarrow \mathbb{R}_{+}^{\alpha}$ is a generalized s-convex function in the second sense, $s \in(0,1]$, $a_{1}, a_{2} \in \mathbb{R}_{+}$which $a_{1}<a_{2}$ and $f \in L^{1}\left(\left[a_{1}, a_{2}\right]\right)$. Then:

(i) $g \in G K_{s}^{2}$ in $[0,1]$. If $f \in G K_{s}^{1}$, then $g \in G K_{s}^{1}$.

(ii) $g\left(\gamma+\frac{1}{2}\right)=g\left(\frac{1}{2}-\gamma\right)$ for all $\gamma=\left[0, \frac{1}{2}\right]$ and $g(\gamma)$ is symmetric about $\gamma=\frac{1}{2}$.

(iii) We have the inequality

$$
g(\gamma) \geq \frac{2^{\alpha(s-1)}}{(\Gamma(1+\alpha))^{2}} A(\gamma) \geq \frac{4^{\alpha(s-1)}}{(\Gamma(1+\alpha))^{2}} f\left(\frac{a_{1}+a_{2}}{2}\right) \quad \text { for } \forall \gamma \in[0,1] .
$$

(iv) We have the inequality

$$
g(\gamma) \leq \min \left\{g_{1}(\gamma), g_{2}(\gamma)\right\}
$$

where

$$
g_{1}(\gamma)=\left[\gamma^{\alpha s}+(1-\gamma)^{\alpha s}\right] \frac{1}{\Gamma(1+\alpha)\left(a_{2}-a_{1}\right)^{\alpha}} a_{1} I_{a_{2}}^{(\alpha)} f\left(x_{1}\right)
$$

and

$$
g_{2}(\gamma)=\left[\frac{\Gamma(1+s \alpha)}{\Gamma(1+(s+1) \alpha)}\right]^{2}\left[f\left(a_{1}\right)+f\left(\gamma a_{1}+(1-\gamma) a_{2}\right)+f\left((1-\gamma) a_{1}+\gamma a_{2}\right)+f\left(a_{2}\right)\right]
$$

for $\forall \gamma \in[0,1]$. 
Proof (i) Take $\left\{\gamma_{1}, \gamma_{2}\right\} \subset[0,1], \gamma_{1}+\gamma_{2}=1, t_{1}, t_{2} \in D$ and $f \in G K_{s}^{2}$, then we have

$$
\begin{aligned}
g\left(\gamma_{1} t_{1}+\gamma_{2} t_{2}\right)= & \frac{1}{\left(a_{2}-a_{1}\right)^{2 \alpha}(\Gamma(1+\alpha))^{2}} \\
& \times \int_{a_{1}}^{a_{2}} \int_{a_{1}}^{a_{2}} f\left(\left(\gamma_{1} t_{1}+\gamma_{2} t_{2}\right) x_{1}+\left(1-\left(\gamma_{1} t_{1}+\gamma_{2} t_{2}\right)\right) x_{2}\right)\left(d x_{1}\right)^{\alpha}\left(d x_{2}\right)^{\alpha} \\
\leq & \frac{1}{\left(a_{2}-a_{1}\right)^{2 \alpha}(\Gamma(1+\alpha))^{2}} \\
& \times \int_{a_{1}}^{a_{2}} \int_{a_{1}}^{a_{2}}\left[\gamma_{1}^{\alpha s} f\left(t_{1} x_{1}+x_{2}-t_{1} x_{2}\right)\right. \\
& \left.+\gamma_{2}^{\alpha s} f\left(t_{2} x_{1}+x_{2}-t_{2} x_{2}\right)\right]\left(d x_{1}\right)^{\alpha}\left(d x_{2}\right)^{\alpha} \\
= & \gamma_{1}^{\alpha s} \frac{1}{\left(a_{2}-a_{1}\right)^{2 \alpha}(\Gamma(1+\alpha))^{2}} \int_{a_{1}}^{a_{2}} \int_{a_{1}}^{a_{2}} f\left(t_{1} x_{1}+x_{2}-t_{1} x_{2}\right)\left(d x_{1}\right)^{\alpha}\left(d x_{2}\right)^{\alpha} \\
& +\gamma_{2}^{\alpha s} \frac{1}{\left(a_{2}-a_{1}\right)^{2 \alpha}(\Gamma(1+\alpha))^{2}} \int_{a_{1}}^{a_{2}} \int_{a_{1}}^{a_{2}} f\left(t_{2} x_{1}+x_{2}-t_{2} x_{2}\right)\left(d x_{1}\right)^{\alpha}\left(d x_{2}\right)^{\alpha} \\
= & \gamma_{1}^{\alpha s} g\left(t_{1}\right)+\gamma_{2}^{\alpha s} g\left(t_{2}\right),
\end{aligned}
$$

which implies that $g \in G K_{s}^{2}$ in $[0,1]$.

(ii) Let $\gamma \in\left[0, \frac{1}{2}\right]$, then

$$
\begin{aligned}
g\left(\gamma+\frac{1}{2}\right)= & \frac{1}{\left(a_{2}-a_{1}\right)^{2 \alpha}(\Gamma(1+\alpha))^{2}} \\
& \times \int_{a_{1}}^{a_{2}} \int_{a_{1}}^{a_{2}} f\left(\left(\gamma+\frac{1}{2}\right) x_{1}+\left(1-\gamma-\frac{1}{2}\right) x_{2}\right)\left(d x_{1}\right)^{\alpha}\left(d x_{2}\right)^{\alpha} \\
= & \frac{1}{\left(a_{2}-a_{1}\right)^{2 \alpha}(\Gamma(1+\alpha))^{2}} \\
& \times \int_{a_{1}}^{a_{2}} \int_{a_{1}}^{a_{2}} f\left(\left(\frac{1}{2}-\gamma\right) x_{1}+\left(\frac{1}{2}+\gamma\right) x_{2}\right)\left(d x_{1}\right)^{\alpha}\left(d x_{2}\right)^{\alpha} \\
= & g\left(\frac{1}{2}-\gamma\right) .
\end{aligned}
$$

$g(\gamma)$ is symmetric about $\gamma=\frac{1}{2}$ because $g(\gamma)=g(1-\gamma)$.

(iii) Let us observe that

$$
g(\gamma)=\frac{1}{(\Gamma(1+\alpha))^{2}} \frac{1}{\left(a_{2}-a_{1}\right)^{\alpha}} \int_{a_{1}}^{a_{2}}\left(\frac{1}{\left(a_{2}-a_{1}\right)^{\alpha}} \int_{a_{1}}^{a_{2}} f\left(\gamma x_{1}+(1-\gamma) x_{2}\right)\right)\left(d x_{1}\right)^{\alpha}\left(d x_{2}\right)^{\alpha} .
$$

Now, since $x_{2}$ is fixed in $\left[a_{1}, a_{2}\right]$, then the function

$$
A_{x_{2}}:[0,1] \rightarrow \mathbb{R}^{\alpha}
$$

can be given by

$$
\begin{aligned}
A_{x_{2}}(\gamma) & =\frac{1}{\left(a_{2}-a_{1}\right)^{\alpha}} \int_{a_{1}}^{a_{2}} f\left(\gamma x_{1}+(1-\gamma) x_{2}\right)\left(d x_{1}\right)^{\alpha} \\
& =\frac{\Gamma(1+\alpha)}{\left(a_{2}-a_{1}\right)^{\alpha}} a_{1} I_{a_{2}}^{(\alpha)} f\left(\gamma x_{1}+(1-\gamma) x_{2}\right) .
\end{aligned}
$$


As it was shown in the proof of Theorem 3.2, for $\gamma \in[0,1]$, we have equality

$$
A_{x_{2}}(\gamma)=\frac{\Gamma(1+\alpha)}{\left(b_{2}-b_{1}\right)^{\alpha}} b_{1} I_{b_{2}}^{(\alpha)} f(m)
$$

where $b_{2}=\gamma a_{2}+(1-\gamma) x_{2}$ and $b_{1}=\gamma a_{1}+(1-\gamma) x_{2}$. By using the generalized HermiteHadamard inequality, we have

$$
\begin{aligned}
\frac{\Gamma(1+\alpha)}{\left(b_{2}-b_{1}\right)^{\alpha}} b_{1} I_{b_{2}}^{(\alpha)} f(m) & \geq 2^{\alpha(s-1)} f\left(\frac{b_{1}+b_{2}}{2}\right) \\
& =2^{\alpha(s-1)} f\left(\gamma \frac{a_{1}+a_{2}}{2}+(1-\gamma) x_{2}\right)
\end{aligned}
$$

for all $\gamma \in(0,1)$ and $x_{2} \in\left[a_{1}, a_{2}\right]$. Integrating on $\left[a_{1}, a_{2}\right]$ over $x_{2}$, we have

$$
g(\gamma) \geq \frac{2^{\alpha(s-1)}}{(\Gamma(1+\alpha))^{2}} A(1-\gamma) \quad \text { for } \forall \gamma \in(0,1) .
$$

Further, since $g(\gamma)=g(1-\gamma)$, then the proof of inequality (8) is done for $\gamma \in(0,1)$. If $\gamma=0$ or $\gamma=1$, then inequality (8) also holds.

(iv) Since $f\left(\gamma x_{1}+(1-\gamma) x_{2}\right) \leq \gamma^{\alpha s} f\left(x_{1}\right)+(1-\gamma)^{\alpha s} f\left(x_{2}\right)$ for all $x_{1}, x_{2} \in\left[a_{1}, a_{2}\right]$ and $\gamma \in[0,1]$, integrating the above inequality on $\left[a_{1}, a_{2}\right]^{2}$, we have

$$
\begin{aligned}
& \frac{1}{\left(a_{2}-a_{1}\right)^{2 \alpha}} \int_{a_{1}}^{a_{2}} \int_{a_{1}}^{a_{2}} f\left(\gamma x_{1}+(1-\gamma) x_{2}\right)\left(d x_{1}\right)^{\alpha}\left(d x_{2}\right)^{\alpha} \\
& \leq \gamma^{\alpha s} \frac{(\Gamma(1+\alpha))^{2}}{\left(a_{2}-a_{1}\right)^{2 \alpha}} \frac{1}{(\Gamma(1+\alpha))^{2}} \int_{a_{1}}^{a_{2}} \int_{a_{1}}^{a_{2}} f\left(x_{1}\right)\left(d x_{1}\right)^{\alpha}\left(d x_{2}\right)^{\alpha} \\
& \quad+(1-\gamma)^{\alpha s} \frac{(\Gamma(1+\alpha))^{2}}{\left(a_{2}-a_{1}\right)^{2 \alpha}} \frac{1}{(\Gamma(1+\alpha))^{2}} \int_{a_{1}}^{a_{2}} \int_{a_{1}}^{a_{2}} f\left(x_{2}\right)\left(d x_{1}\right)^{\alpha}\left(d x_{2}\right)^{\alpha} \\
& =\gamma^{\alpha s} \frac{\Gamma(1+\alpha)}{\left(a_{2}-a_{1}\right)^{\alpha}} a_{1} I_{a_{2}}^{(\alpha)} f\left(x_{1}\right)+(1-\gamma)^{\alpha s} \frac{\Gamma(1+\alpha)}{\left(a_{2}-a_{1}\right)^{\alpha} a_{1} I_{a_{2}}^{(\alpha)} f\left(x_{2}\right)} \\
& =\left(\gamma^{\alpha s}+(1-\gamma)^{\alpha s}\right) \frac{\Gamma(1+\alpha)}{\left(a_{2}-a_{1}\right)^{\alpha}} a_{1} I_{a_{2}}^{(\alpha)} f\left(x_{1}\right) .
\end{aligned}
$$

The proof of the first part in (9) is done.

By the second part of the generalized Hermite-Hadamard inequality, we obtain

$$
\begin{aligned}
A_{x_{2}}(\gamma) & =\frac{\Gamma(1+\alpha)}{\left(b_{2}-b_{1}\right)^{\alpha}} b_{1} I_{b_{2}}^{(\alpha)} f(m) \\
& \leq \frac{\Gamma(1+s \alpha) \Gamma(1+\alpha)}{\Gamma(1+(s+1) \alpha)}\left(f\left(\gamma a_{1}+(1-\gamma) x_{2}\right)+f\left(\gamma a_{2}+(1-\gamma) x_{2}\right)\right)
\end{aligned}
$$

where $b_{2}=\gamma a_{2}+(1-\gamma) x_{2}$ and $b_{1}=\gamma a_{1}+(1-\gamma) x_{2}, \gamma \in[0,1]$. Integrating this inequality on $\left[a_{1}, a_{2}\right]$ over $x_{2}$, then

$$
\begin{aligned}
g(\gamma) \leq & \frac{\Gamma(1+s \alpha)}{\Gamma(1+\alpha) \Gamma(1+(s+1) \alpha)}\left[\frac{\Gamma(1+\alpha)}{\left(a_{2}-a_{1}\right)^{\alpha}} a_{1} I_{a_{2}}^{(\alpha)} f\left(\gamma a_{1}+(1-\gamma) x_{2}\right)\right. \\
& \left.+\frac{\Gamma(1+\alpha)}{\left(a_{2}-a_{1}\right)^{\alpha}} a_{1} I_{a_{2}}^{(\alpha)} f\left(\gamma a_{2}+(1-\gamma) x_{2}\right)\right] .
\end{aligned}
$$


A simple calculation shows that

$$
\begin{aligned}
& \frac{\Gamma(1+\alpha)}{\left(a_{2}-a_{1}\right)^{\alpha}} a_{1} I_{a_{2}}^{(\alpha)} f\left(\gamma a_{2}+(1-\gamma) x_{2}\right) \\
& \quad=\frac{\Gamma(1+\alpha)}{\left(c_{2}-c_{1}\right)^{\alpha}} c_{1} I_{c_{2}}^{(\alpha)} f(m) \\
& \quad \leq \frac{\Gamma(1+s \alpha) \Gamma(1+\alpha)}{\Gamma(1+(s+1) \alpha)}\left[f\left(c_{1}\right)+f\left(c_{2}\right)\right] \\
& \quad=\frac{\Gamma(1+s \alpha) \Gamma(1+\alpha)}{\Gamma(1+(s+1) \alpha)}\left[f\left(a_{2}\right)+f\left(\gamma a_{2}+(1-\gamma) a_{1}\right)\right],
\end{aligned}
$$

where $c_{2}=a_{2}, c_{1}=\gamma a_{2}+(1-\gamma) a_{1}$ and $\gamma \in(0,1)$. Similarly, for $\gamma \in(0,1)$,

$$
\begin{aligned}
& \frac{\Gamma(1+\alpha)}{\left(a_{2}-a_{1}\right)^{\alpha}} a_{1} I_{a_{2}}^{(\alpha)} f\left(\gamma a_{1}+(1-\gamma) x_{2}\right) \\
& \quad \leq \frac{\Gamma(1+s \alpha) \Gamma(1+\alpha)}{\Gamma(1+(s+1) \alpha)}\left[f\left(a_{1}\right)+f\left(\gamma a_{1}+(1-\gamma) a_{2}\right)\right] .
\end{aligned}
$$

Then

$$
g(\gamma) \leq\left[\frac{\Gamma(1+s \alpha)}{\Gamma(1+(s+1) \alpha)}\right]^{2}\left[f\left(a_{1}\right)+f\left(\gamma a_{1}+(1-\gamma) a_{2}\right)+f\left((1-\gamma) a_{1}+\gamma a_{2}\right)+f\left(a_{2}\right)\right] .
$$

If $\gamma=0$ or $\gamma=1$, then this inequality also holds.

Remark 3.3 If $\alpha=1$ in the above theorem, then

$$
g(\gamma)=\frac{1}{\left(a_{2}-a_{1}\right)^{2}} \int_{a_{1}}^{a_{2}} \int_{a_{1}}^{a_{2}} f\left(\gamma x_{1}+(1-\gamma) x_{2}\right)\left(d x_{1}\right)\left(d x_{2}\right), \quad \gamma \in[0,1]
$$

and

$$
\begin{aligned}
g(\gamma) \leq & \min \left\{\left[\gamma^{s}+(1-\gamma)^{s}\right] \frac{1}{\left(a_{2}-a_{1}\right)} \int_{a_{1}}^{a_{2}} f\left(x_{1}\right)\left(d x_{1}\right),\right. \\
& \left.\frac{1}{(1+s)^{2}}\left[f\left(a_{1}\right)+f\left(\gamma a_{1}+(1-\gamma) a_{2}\right)+f\left((1-\gamma) a_{1}+\gamma a_{2}\right)+f\left(a_{2}\right)\right]\right\} .
\end{aligned}
$$

Theorem 3.4 Let us consider that a sum of A belongs to $G K_{s}^{2}$,

$$
A=\sum_{i=1}^{n} a_{i}(\gamma),
$$

where

$$
a_{i}(\gamma)=\frac{1}{(\Gamma(1+\alpha))^{2}} \int_{a_{1}}^{a_{2}} \int_{a_{1}}^{a_{2}} f_{i}\left(\gamma x_{1}+(1-\gamma) x_{2}\right)\left(d x_{1}\right)^{\alpha}\left(d x_{2}\right)^{\alpha},
$$

then

(i) $\sup (A)=2^{\alpha} \sum_{i=1}^{n} a_{i}(0)=2^{\alpha} \sum_{i=1}^{n} a_{i}(1)$,

(ii) $A$ is symmetric about $\gamma=\frac{1}{2}$,

(iii) $A \in G K_{s}^{2}$. 
Proof (i)

$$
\begin{aligned}
a_{i}(\gamma) \leq & \gamma^{\alpha s} \frac{1}{(\Gamma(1+\alpha))^{2}} \int_{a_{1}}^{a_{2}} \int_{a_{1}}^{a_{2}} f_{i}\left(x_{1}\right)\left(d x_{1}\right)^{\alpha}\left(d x_{2}\right)^{\alpha} \\
& +(1-\gamma)^{\alpha s} \frac{1}{(\Gamma(1+\alpha))^{2}} \int_{a_{1}}^{a_{2}} \int_{a_{1}}^{a_{2}} f_{i}\left(x_{2}\right)\left(d x_{1}\right)^{\alpha}\left(d x_{2}\right)^{\alpha}, \quad \forall i .
\end{aligned}
$$

Since $f_{i}$ are generalized $s$-convex functions, we get

$$
\begin{aligned}
a_{i}(\gamma) & \leq\left(\gamma^{\alpha s}+(1-\gamma)^{\alpha s}\right) \frac{1}{(\Gamma(1+\alpha))^{2}} \int_{a_{1}}^{a_{2}} \int_{a_{1}}^{a_{2}} f_{i}\left(x_{1}\right)\left(d x_{1}\right)^{\alpha}\left(d x_{2}\right)^{\alpha} \\
& \leq \frac{2^{\alpha}}{(\Gamma(1+\alpha))^{2}} \int_{a_{1}}^{a_{2}} \int_{a_{1}}^{a_{2}} f_{i}\left(x_{1}\right)\left(d x_{1}\right)^{\alpha}\left(d x_{2}\right)^{\alpha} \\
& =2^{\alpha} a_{i}(0)=2^{\alpha} a_{i}(1) .
\end{aligned}
$$

(ii) $a_{i}(\gamma)$ is symmetric about $\gamma=\frac{1}{2}$ since $a_{i}(\gamma)=a_{i}(1-\gamma), \forall i$.

Then $A(1-\gamma)=A(\gamma)$ and $A$ also is.

(iii) Since $a_{i}\left(\gamma x_{1}+(1-\gamma) x_{2}\right) \leq \gamma^{\alpha s} a_{i}\left(x_{1}\right)+(1-\gamma)^{\alpha s} a_{i}\left(x_{2}\right)$, then

$$
\begin{aligned}
A\left(\gamma x_{1}+(1-\gamma) x_{2}\right) & =\sum_{i=1}^{n} a_{i}\left(\gamma x_{1}+(1-\gamma) x_{2}\right) \\
& \leq \gamma^{\alpha s} \sum_{i=1}^{n} a_{i}\left(x_{1}\right)+(1-\gamma)^{\alpha s} \sum_{i=1}^{n} a_{i}\left(x_{2}\right) \\
& =\gamma^{\alpha s} A\left(x_{1}\right)+(1-\gamma)^{\alpha s} A\left(x_{2}\right),
\end{aligned}
$$

that is, $A \in G K_{s}^{2}$.

\section{Applications to special means}

We now consider the applications of our theorems to the following generalized means:

$$
\begin{aligned}
& A\left(a_{1}, a_{2}\right)=\frac{a_{1}^{\alpha}+a_{2}^{\alpha}}{2^{\alpha}}, \quad a_{1}, a_{2} \geq 0, \\
& K\left(a_{1}, a_{2}\right)=\left(\frac{a_{1}^{2 \alpha}+a_{2}^{2 \alpha}}{2^{\alpha}}\right)^{\frac{1}{2}}, \quad a_{1}, a_{2} \geq 0
\end{aligned}
$$

and

$$
G\left(a_{1}, a_{2}\right)=\left(a_{1}^{\alpha} a_{2}^{\alpha}\right)^{\frac{1}{2}}, \quad a_{1}, a_{2} \geq 0 .
$$

In [23], the following example is given.

Let $0<s<1$ and $a_{1}^{\alpha}, a_{2}^{\alpha}, a_{3}^{\alpha} \in \mathbb{R}^{\alpha}$. Define, for $x \in \mathbb{R}_{+}$,

$$
f(n)= \begin{cases}a_{1}^{\alpha}, & n=0, \\ a_{2}^{\alpha} n^{s \alpha}+a_{3}^{\alpha}, & n>0 .\end{cases}
$$

If $a_{2}^{\alpha} \geq 0^{\alpha}$ and $0^{\alpha} \leq a_{3}^{\alpha} \leq a_{1}^{\alpha}$, then $f \in G K_{s}^{2}$. 
Proposition 4.1 Let $a_{1}, a_{2} \in \mathbb{R}_{+}, a_{1}<a_{2}$ and $a_{2}-a_{1} \leq 1$, then the following inequalities hold:

$$
\begin{aligned}
& \frac{\Gamma(1+2 \alpha)}{\Gamma(1+\alpha) \Gamma(1+3 \alpha)}\left[K^{2}\left(a_{1}, a_{2}\right)+\frac{1}{2^{\alpha}} G^{2}\left(a_{1}, a_{2}\right)\right] \geq 2^{\alpha}\left[\frac{\Gamma(1+\alpha)}{\Gamma(1+2 \alpha)}\right]^{2} A^{2}\left(a_{1}, a_{2}\right), \\
& {\left[\left(1-\gamma+\gamma^{2}\right)^{\alpha} K^{2}\left(a_{1}, a_{2}\right)+\gamma^{\alpha}(1-\gamma)^{\alpha} G^{2}\left(a_{1}, a_{2}\right)\right]} \\
& \quad-\frac{\gamma^{2 \alpha}+(1-\gamma)^{\alpha}}{2^{\alpha} \Gamma(1+3 \alpha)}\left[\frac{\Gamma(1+2 \alpha)}{\Gamma(1+\alpha)}\right]^{2}\left[K^{2}\left(a_{1}, a_{2}\right)+\frac{1}{2^{\alpha}} G^{2}\left(a_{1}, a_{2}\right)\right] \\
& \quad \geq 2^{\alpha} \gamma^{\alpha}(1-\gamma)^{\alpha} A^{2}\left(a_{1}, a_{2}\right) .
\end{aligned}
$$

Proof If $f \in G K_{s}^{2}$ on $\left[a_{1}, a_{2}\right]$ for some $\gamma \in[0,1]$ and $s \in(0,1]$, then, in Theorem 3.3, if $f:[0,1] \rightarrow\left[0^{\alpha}, 1^{\alpha}\right], f(x)=x^{2 \alpha}$, where $x \in\left[a_{1}, a_{2}\right]$ and $s=1$, so

$$
\begin{aligned}
& \frac{1}{(\Gamma(1+\alpha))^{2}} \frac{1}{\left(a_{2}-a_{1}\right)^{2 \alpha}} \int_{a_{1}}^{a_{2}} \int_{a_{1}}^{a_{2}}\left(\gamma x_{1}+(1-\gamma) x_{2}\right)^{2 \alpha}\left(d x_{1}\right)^{\alpha}\left(d x_{2}\right)^{\alpha} \\
& =\left(\gamma^{2 \alpha}+(1-\gamma)^{2 \alpha}\right) \frac{\Gamma(1+2 \alpha)}{\Gamma(1+\alpha) \Gamma(1+3 \alpha)}\left(a_{2}^{2 \alpha}+a_{1}^{\alpha} a_{2}^{\alpha}+a_{1}^{2 \alpha}\right) \\
& \quad+2^{\alpha} \gamma^{\alpha}(1-\gamma)^{\alpha}\left[\frac{\Gamma(1+\alpha)}{\Gamma(1+2 \alpha)}\right]^{\alpha}\left(a_{2}^{\alpha}+a_{1}^{\alpha}\right)^{2} .
\end{aligned}
$$

Then, by Theorem 3.3, we get

$$
\frac{\Gamma(1+2 \alpha)}{\Gamma(1+\alpha) \Gamma(1+3 \alpha)}\left(a_{2}^{2 \alpha}+a_{1}^{2 \alpha}+a_{1}^{\alpha} a_{2}^{\alpha}\right) \geq\left[\frac{\Gamma(1+\alpha)}{\Gamma(1+2 \alpha)}\right]^{2}\left(a_{2}^{\alpha}+a_{1}^{\alpha}\right)^{2} .
$$

Then we obtain inequality (10).

By applying Theorem 3.3, we obtain inequality (11) as follows:

$$
\begin{aligned}
& {\left[\left(1-\gamma+\gamma^{2}\right)^{\alpha}\left(\frac{a_{2}^{2 \alpha}+a_{1}^{2 \alpha}}{2^{\alpha}}\right)+\gamma^{\alpha}(1-\gamma)^{\alpha} a_{1}^{\alpha} a_{2}^{\alpha}\right]} \\
& \quad-\frac{\gamma^{2 \alpha}+(1-\gamma)^{2 \alpha}}{2^{\alpha} \Gamma(1+3 \alpha)}\left[\frac{\Gamma(1+2 \alpha)}{\Gamma(1+\alpha)}\right]^{2}\left[\left(\frac{a_{2}^{2 \alpha}+a_{1}^{2 \alpha}}{2^{\alpha}}\right)+\frac{1}{2^{\alpha}} a_{1}^{\alpha} a_{2}^{\alpha}\right] \\
& \quad \geq 2^{\alpha} \gamma^{\alpha}(1-\gamma)^{\alpha}\left(\frac{a_{2}^{\alpha}+a_{1}^{\alpha}}{2^{\alpha}}\right)^{2} .
\end{aligned}
$$

\section{Conclusion}

In this article, we have established some new integral inequalities of generalized HermiteHadamard type for generalized $s$-convex functions in the second sense on fractal sets $\mathbb{R}^{\alpha}$, $0<\alpha<1$. In particular, our results extend some important inequalities in a classical situation; when $\alpha=1$, some relationships between these inequalities and the classical inequalities have been established. Finally, we have also given some applications for these inequalities on fractal sets. 


\section{Author details}

'Department of Mathematics, Institute for Mathematical Research, University Putra Malaysia, Serdang, Malaysia.

${ }^{2}$ Department of Mathematics, University Putra Malaysia, Serdang, Malaysia.

\section{Acknowledgements}

The authors would like to thank the referees for valuable suggestions and comments, which helped the authors to improve this article substantially.

\section{Received: 19 June 2015 Accepted: 15 September 2015 Published online: 24 September 2015}

\section{References}

1. Kılıçman, A, Saleh, W: Some inequalities for generalized s-convex functions. JP J. Geom. Topol. 17, 63-82 (2015)

2. Ruel, JJ, Ayres, MP: Jensen's inequality predicts effects of environmental variation. Trends Ecol. Evol. 14(9), 361-366 (1999)

3. Li, M, Wang, J, Wei, W: Some fractional Hermite-Hadamard inequalities for convex and Godunova-Levin functions. Facta Univ., Ser. Math. Inform. 30(2), 195-208 (2015)

4. Liu, W: Ostrowski type fractional integral inequalities for MT-convex functions. Miskolc Math. Notes 16(1), 249-256 (2015)

5. Lin, Z, Wang, JR, Wei, W: Fractional Hermite-Hadamard inequalities through $r$-convex functions via power means. Facta Univ., Ser. Math. Inform. 30(2), 129-145 (2015)

6. Liu, W: New integral inequalities involving beta function via P-convexity. Miskolc Math. Notes 15(2), $585-591$ (2014)

7. Budak, H, Sarikaya, MZ: Some new generalized Hermite-Hadamard inequality for generalized convex function and applications. RGMIA Res. Rep. Collect. 18, 82 (2015)

8. Dragomir, SS, Fitzpatrick, S: The Hadamard inequalities for s-convex functions in the second sense. Demonstr. Math. 32(4), 687-696 (1999)

9. Golmankhaneh, AK, Baleanu, D: On a new measure on fractals. J. Inequal. Appl. 2013, 552 (2013)

10. Kolwankar, KM, Gangal, AD: Local fractional calculus: a calculus for fractal space-time. In: Fractals: Theory and Applications in Engineering, pp. 171-181. Springer, London (1999)

11. Yang, Y-J, Baleanu, D, Yang, X-J: Analysis of fractal wave equations by local fractional Fourier series method. Adv. Math Phys. 2013, Article ID 632309 (2013)

12. Noor, MA, Noor, Kl, Awan, MU, Khan, S: Fractional Hermite-Hadamard inequalities for some new classes of Godunova-Levin functions. Appl. Math. Inf. Sci. 8(6), 2865-2872 (2014)

13. Liu, W: Some Ostrowski type inequalities via Riemann-Liouville fractional integrals for $h$-convex functions. J. Comput. Anal. Appl. 16(5), 998-1004 (2014)

14. Erden, S, Sarikaya, MZ: Generalized Bullen type inequalities for local fractional integrals and its applications. RGMIA Res. Rep. Collect. 18, 81 (2015)

15. Budak, H, Sarikaya, MZ, Yildirim, H: New inequalities for local fractional integrals. RGMIA Res. Rep. Collect. 18, $88(2015)$

16. Babakhani, A, Daftardar-Gejji, V: On calculus of local fractional derivatives. J. Math. Anal. Appl. 270(1), 66-79 (2002)

17. Carpinteri, A, Chiaia, B, Cornetti, P: Static-kinematic duality and the principle of virtual work in the mechanics of fractal media. Comput. Methods Appl. Mech. Eng. 191(1-2), 3-19 (2001)

18. Zhao, Y, Cheng, DF, Yang, $X J$ : Approximation solutions for local fractional Schrödinger equation in the one-dimensional Cantorian system. Adv. Math. Phys. 2013, Article ID 291386 (2013)

19. Yang, XJ: Advanced Local Fractional Calculus and Its Applications. World Science Publisher, New York (2012)

20. Yang, XJ, Baleanu, D, Machado, JAT: Mathematical aspects of Heisenberg uncertainty principle within local fractional Fourier analysis. Bound. Value Probl. 2013(1), 131 (2013)

21. Yang, AM, Chen, ZS, Srivastava, HM, Yang, XJ: Application of the local fractional series expansion method and the variational iteration method to the Helmholtz equation involving local fractional derivative operators. Abstr. Appl. Anal. 2013, Article ID 259125 (2013)

22. Yang, XJ, Baleanu, D, Khan, Y, Mohyud-Din, ST: Local fractional variational iteration method for diffusion and wave equations on Cantor sets. Rom. J. Phys. 59(1-2), 36-48 (2014)

23. Mo, H, Sui, X: Generalized s-convex functions on fractal sets. Abstr. Appl. Anal. 2014 Article ID 254731 (2014)

24. Mo, H, Sui, X, Yu, D: Generalized convex functions on fractal sets and two related inequalities. Abstr. Appl. Anal. 2014, Article ID 636751 (2014)

25. Hörmander, L: Notions of Convexity. Birkhäuser, Basel (1994)

26. Mo, H, Sui, X: Hermite-Hadamard type inequalities for generalized s-convex functions on real linear fractal set $\mathbb{R}^{\alpha}$ $(0<\alpha<1)$ (2015). arXiv:1506.07391 